\title{
A DOMAIN DECOMPOSITION METHOD FOR ADVECTION-DIFFUSION PROCESSES WITH APPLICATION TO BLOOD SOLUTES*
}

\author{
ALFIO QUARTERONI ${ }^{\dagger}$, ALESSANDRO VENEZIANI $^{\ddagger}$, AND PAOLO ZUNINO $§$
}

\begin{abstract}
In the present paper we consider a heterogeneous model for the dynamics of a blood solute both in the vascular lumen and inside the arterial wall. In the lumen, we consider an advection-diffusion equation, where the convective field is provided by the velocity of blood, which is in turn obtained by solving the Navier-Stokes equations. Inside the arterial wall we consider a pure diffusive dynamics. Since the endothelial layer at the interface between the lumen and the wall acts as a permeable membrane, whose permeability depends on the shear rate exerted by the blood, the solute concentration is discontinuous across this membrane. A possible approach for the numerical study of this kind of problem is inspired by domain decomposition techniques. In particular, we introduce a splitting in the computation and alternate the solution of the advectiondiffusion equation in the lumen with that of the diffusion equation in the wall. We set up an efficient iterative method, based on a suitable reformulation of the problem in terms of a Steklov-Poincaré interface equation. This formulation is a nonstandard one because of the concentration discontinuity at the lumen-wall interface and plays a key role in the proof of convergence of our method. In particular, we prove that the convergence rate performed by the proposed method is independent of the finite element space discretization and provides a criterion for the selection of an acceleration parameter.
\end{abstract}

Several numerical results, referred to as biomedical applications, support our theoretical conclusions and illustrate the efficiency of this algorithm.

Key words. heterogeneous models, domain decomposition techniques, Steklov-Poincaré operators, advection-diffusion equations, finite elements

AMS subject classifications. 76D05, 76Z05, 76R50, 76M10, 65M60, 65Y99

PII. S1064827500375722

1. Introduction. We consider the numerical treatment of a model for the dynamics of blood solutes which was introduced in [14]. This model is based on an advection-diffusion equation describing the solute dynamics in the vascular lumen, the convective field being provided by the blood velocity. This equation is coupled with a pure diffusive model accounting for the solute dynamics inside the arterial wall, where convection is negligible. The two subdomains (namely the lumen and the wall) are physically separated by the endothelial layer, which acts as a selective permeable membrane. The interface equation matching the two subproblems in fact follows from the specific nature of this membrane. It is worthwhile noticing that, due to the presence of this membrane, the two concentrations fail to match continuously at the interface.

*Received by the editors July 25, 2000; accepted for publication (in revised form) November 1, 2001; published electronically February 27, 2002. This work was supported by MURST research contracts and the GNIM-INDAM project "Modelling of Mass Transport Processes in Heterogeneous Media."

http://www.siam.org/journals/sisc/23-6/37572.html

†Dipartimento di Matematica "F. Brioschi," Politecnico di Milano, Piazza L. da Vinci 32, I-20133 Milan, Italy and Département de Mathématiques, École Polytechnique Fédérale de Lausanne, CH-1015, Lausanne, Switzerland (Alfio.Quarteroni@epfl.ch).

‡Dipartimento di Matematica "F. Brioschi," Politecnico di Milano, Piazza L. da Vinci 32, I-20133 Milan, Italy (ales@mate.polimi.it).

§Département de Mathématiques, École Polytechnique Fédérale de Lausanne, CH-1015, Lausanne, Switzerland (Paolo.Zunino@epfl.ch). 
The well posedness of this model, coupled with the Navier-Stokes equations for the description of the blood velocity and pressure fields, has been analyzed in [14]. The interest for this problem stems from the consideration that, in some cases, the cause of widespread pathologies of the vascular system has been related to specific features of the blood flow in a diseased district as well as to the influence of the flow pattern on the transfer processes of solutes between blood and walls (see, e.g., [1], [7], [15], [2], [3]). In [2], [3], for example, a more complex physiological model has been introduced; however, so far it has been tested only on simple two-dimensional (2D) geometries. On the other hand, more realistic three-dimensional (3D) calculations have been pursued in [7]; however, in this case the model does not include the arterial wall as a domain, per se; rather it surrogates the artery wall by prescribing a Dirichlet boundary condition for the concentration. In this paper, our aim is to set up efficient numerical methods for solving heterogeneous problems featuring a discontinuous solution. Although stemming from the specific application at hand, the interest of these methods goes beyond it as they can be applied to any system of advection-diffusion equations which models the transfer of mass between heterogeneous media through permeable membranes.

In the numerical analysis of these kinds of problems, a monolithic solver is typically adopted. Our approach is based on domain decomposition methods, solving alternatively the different problems in the different subdomains (iterative substructuring approach). In such a way, the discontinuity at the interface is accounted for naturally. The general theory underlying this approach has been developed in [13, Chapters 1 and 4]. However, the discontinuity of the solution in this specific application makes the convergence analysis of the method "nonstandard." In particular, we will focus on the choice of convenient preconditioned iterative techniques and the proof of convergence in the very general and abstract framework provided by the SteklovPoincare operator theory. In a forthcoming paper, we will consider the extension of these techniques to the more realistic models considered in [2], [3].

The paper is organized as follows. In section 2 we provide a brief introduction to the heterogeneous model and recall the well posedness results obtained in [14]. In section 3 we provide a reinterpretation of the problem in terms of a Steklov-Poincaré interface equation. This reformulation is not a mere extension of techniques adopted in other contexts, due to the presence of discontinuous solutions. In section 4, we introduce an iterative method for the solution of the problem by solving successively the solute dynamics in the lumen and in the wall. Starting from the Steklov-Poincaré reformulation of the interface problem, we show that this method can be regarded as a particular Richardson preconditioned method for the interface problem. Then, we carry out the convergence analysis of this method; we identify an optimal preconditioner associated to it and propose various generalizations based on the (flexible) preconditioned GMRES method.

The numerical results presented refer to biomedical applications and illustrate the efficiency of our schemes (section 5).

1.1. Some notation. Let $\Omega \subset \mathbb{R}^{d}(d=2,3)$ be a physical bounded domain and $\mathbf{x} \in \Omega$. We denote by $L^{2}(\Omega)$ the Hilbert space of square integrable functions in $\Omega$. The scalar product in $L^{2}(\Omega)$ is denoted by $(\cdot, \cdot)$ and the related norm by $\|\cdot\|_{L^{2}(\Omega)}$. The space of essentially bounded functions in $\Omega$ is denoted by $L^{\infty}(\Omega)$. The Sobolev space of functions, whose first (distributional) derivatives belong to $L^{2}(\Omega)$, is denoted by $H^{1}(\Omega)$ and its norm by $\|\cdot\|_{H^{1}(\Omega)}$.

If $\Sigma \subset \partial \Omega$ is open and nonempty, then the space of functions defined on $\Sigma$ which 
are traces of functions belonging to $H^{1}(\Omega)$ is indicated by $H^{1 / 2}(\Sigma)$. We recall (see [5]) that the trace operator $\gamma: H^{1}(\Omega) \rightarrow H^{1 / 2}(\Sigma)$ is surjective and continuous and there exists an injective, linear, and continuous map $\mathcal{L}: H^{1 / 2}(\Sigma) \rightarrow H^{1}(\Omega)$ called lifting such that $\lambda=\gamma \mathcal{L} \lambda$ for all $\lambda \in H^{1 / 2}(\Sigma)$. In particular, denoting by $\phi$ a function in $H^{1}(\Omega)$ and $\gamma \phi$ its trace on $\Sigma$, the following trace inequality holds:

$$
\exists \beta_{t}>0: \quad\|\gamma \phi\|_{H^{1 / 2}(\Sigma)} \leq \beta_{t}\|\phi\|_{H^{1}(\Omega)} \quad \forall \phi \in H^{1 / 2}(\Sigma) .
$$

We denote by $H_{\Sigma}^{1}(\Omega)$ the subspace of $H^{1}(\Omega)$ of the functions that have null traces on $\Sigma$. In particular, we adopt the usual notation $H_{0}^{1}(\Omega)=H_{\partial \Omega}^{1}(\Omega)$. In $H_{\Sigma}^{1}(\Omega)$ the following Poincaré inequality holds:

$$
\exists \alpha>0: \quad\|\phi\|_{L^{2}(\Omega)} \leq \alpha\|\nabla \phi\|_{L^{2}(\Omega)} \quad \forall \phi \in H_{\Sigma}^{1}(\Omega) .
$$

If $\Gamma$ denotes a $(d-1)$-dimensional manifold in $\bar{\Omega}$ with $\Gamma \cap \Sigma \neq \emptyset$, the trace of $u \in H_{\Sigma}^{1}(\Omega)$ on $\Gamma$ belongs to a subspace of $H^{1 / 2}(\Gamma)$, usually denoted by $H_{00}^{1 / 2}(\Gamma)$. To simplify our notation we will set $\Lambda=H_{00}^{1 / 2}(\Gamma)$, and $\Lambda^{\prime}$ will denote its dual (see [5]). We remark that if $\zeta$ is any positive function in $L^{2}(\Sigma)$, the following definition makes sense:

$$
(\rho, \lambda)_{\zeta}=\int_{\Sigma} \zeta \rho \lambda=(\sqrt{\zeta} \lambda, \sqrt{\zeta} \rho) \quad \forall \lambda, \rho \in H^{1 / 2}(\Sigma) .
$$

Indeed, by the Sobolev embedding theorem, $\lambda$ and $\rho$ belong to $L^{4}(\Sigma)$; hence $\lambda \rho$ belongs to $L^{2}(\Sigma)$. Denoting by $\mathcal{L} \lambda$ and $\mathcal{L} \rho$ any continuous lifting of $\lambda$ and $\rho$ from $\Sigma$ to $\Omega$, it follows that

$$
\left|(\rho, \lambda)_{\zeta}\right| \leq \beta_{e}^{2}\|\zeta\|_{L^{2}(\Sigma)}\|\lambda\|_{H^{1 / 2}(\Sigma)}\|\rho\|_{H^{1 / 2}(\Sigma)} \leq \beta^{2}\|\mathcal{L} \lambda\|_{H^{1}(\Omega)}\|\mathcal{L} \rho\|_{H^{1}(\Omega)},
$$

with $\beta^{2}=\beta_{e}^{2} \beta_{t}^{2}\|\zeta\|_{L^{2}(\Sigma)}$, where $\beta_{e}$ is the embedding constant of $H^{1 / 2}(\Sigma)$ in $L^{4}(\Sigma)$ and $\beta_{t}$ is the constant of the trace inequality (1.1). Finally, we set

$$
\|\lambda\|_{\zeta}^{2}=(\lambda, \lambda)_{\zeta}=\|\sqrt{\zeta} \lambda\|_{L^{2}(\Sigma)}^{2} .
$$

For space-time functions $v: \Omega \times(0, T) \rightarrow \mathbb{R}$, for all real $q$ and $s=0,1$, we introduce the space

$$
L^{q}\left(0, T ; H^{s}(\Omega)\right) \equiv\left\{v:(0, T) \rightarrow H^{s} \mid v(t) \text { is measurable, } \int_{0}^{T}\|v(t)\|_{H^{s}(\Omega)}^{q} d t<\infty\right\}
$$

endowed with the norm

$$
\|v\|_{L^{q}\left(0, T ; H^{s}(\Omega)\right)} \equiv\left(\int_{0}^{T}\|v(t)\|_{H^{s}(\Omega)}^{q} d t\right)^{1 / q}
$$

2. Problem formulation. Let us consider a specific vascular district $\Omega \subset \mathbb{R}^{d}$ $(d=2,3)$, composed by a lumen or a fluid subdomain $\Omega_{f}$ and a structure or wall subdomain $\Omega_{w}$. Their interface $\Gamma$ belongs to $\mathbb{R}^{d-1}$ (see Figure 2.1).

The artificial sections delimiting the district proximally and distally with respect to the heart will be denoted by $\Gamma_{u p}$ and $\Gamma_{d w}$, respectively. For $\mathbf{x} \in \Omega$ and $t>0$ we denote by $\mathbf{u}(\mathbf{x}, t)$ the velocity of the blood and by $P(\mathbf{x}, t)$ its pressure. $C_{f}(\mathbf{x}, t)$ and $C_{w}(\mathbf{x}, t)$ denote the concentrations of the solute in the lumen $\Omega_{f}$ and in the wall $\Omega_{w}$, respectively. We assume the blood to be an incompressible Newtonian fluid (which is a realistic assumption in large and medium vessels - see, e.g., [11]) within rigid walls. Then, the blood motion is described by the Navier-Stokes incompressible equations, obtained by the momentum and mass conservation principles. The initial-boundary values problem we are going to consider for the blood dynamics therefore reads 


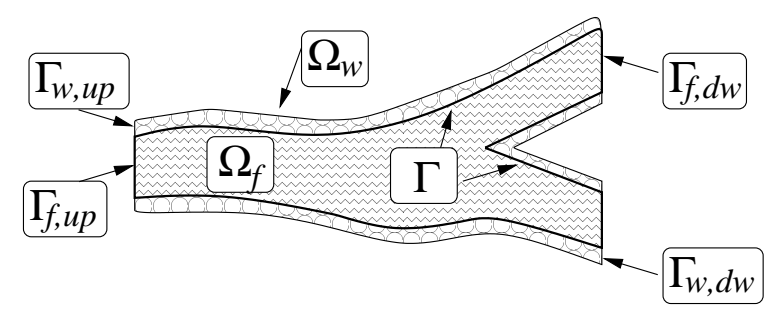

FIG. 2.1. Computational domain representing a $2 D$ section of a vascular district featuring the lumen $\Omega_{f}$ and the wall $\Omega_{w}$.

$$
\begin{aligned}
& \rho \frac{\partial \mathbf{u}}{\partial t}+\rho(\mathbf{u} \cdot \nabla) \mathbf{u}-\nu \Delta \mathbf{u}+\nabla P=\mathbf{f} \quad \mathbf{x} \in \Omega_{f}, \quad t>0, \\
& \nabla \cdot \mathbf{u}=0 \quad \mathbf{x} \in \Omega_{f}, \quad t>0, \\
& \mathbf{u}=\mathbf{b} \quad \text { on } \quad \Gamma_{f, u p}, \quad t>0, \quad \mathbf{u}=\mathbf{0} \quad \text { on } \quad \Gamma, \quad t>0, \\
& P \mathbf{n}-\rho \nu \nabla \mathbf{u} \cdot \mathbf{n}=P_{e x t} \mathbf{n} \quad \text { on } \quad \Gamma_{f, d w}, \quad t>0, \\
& \mathbf{u}(\mathbf{x}, t)=\mathbf{u}_{0} \quad \text { with } \quad \nabla \cdot \mathbf{u}_{0}=0, \quad \mathbf{x} \in \Omega_{f}, \quad t=0 .
\end{aligned}
$$

In (2.1) we suppose that the boundary $\partial \Omega_{f}$ of the computational domain of the fluid part of the problem is split into the interface with the wall $\Gamma$, the upstream or proximal part $\Gamma_{f, u p}$ (where we prescribe the velocity field), and the downstream or distal one, $\Gamma_{f, d w}$ (where the normal fluid stress is assigned). We are also assuming that the density of the blood $\rho_{b}$ as well as the viscosity $\nu$ are constant; the physiological range of these parameters is discussed in section 5 .

The dynamics of solutes is described by an advection-diffusion process. In the lumen, the convective field is provided by the blood velocity, while in the wall, because of the very low velocity of the solvent, the advection is negligible (see [8]). The interface $\Gamma$ can be regarded as a permeable membrane whose permeability $\zeta$ is a positive function of the shear stress $\sigma$ exerted by the blood on the wall (see [15]). More precisely, the solute flux through $\Gamma$ is proportional to the difference of concentration between lumen and wall. All these considerations lead to the following model for the solute concentrations for all $t>0$ :

$$
\left\{\begin{array}{l}
\frac{\partial C_{f}}{\partial t}-\mu_{f} \Delta C_{f}+\mathbf{u} \cdot \nabla C_{f}=s_{f} \text { in } \Omega_{f}, \\
\frac{\partial C_{w}}{\partial t}-\mu_{w} \Delta C_{w}=s_{w} \text { in } \Omega_{w}, \\
\mu_{f} \frac{\partial C_{f}}{\partial \mathbf{n}_{f}}+\zeta\left(C_{f}-C_{w}\right)=0 \text { on } \Gamma, \\
\mu_{w} \frac{\partial C_{w}}{\partial \mathbf{n}_{w}}+\zeta\left(C_{w}-C_{f}\right)=0 \text { on } \Gamma, \\
C_{f}=C_{f, u p} \text { on } \Gamma_{f, u p}, \quad C_{w}=C_{w, u p} \text { on } \Gamma_{w, u p}, \\
\mu_{f} \frac{\partial C_{f}}{\partial \mathbf{n}_{f}}=0 \quad \text { on } \Gamma_{f, d w}, \quad \mu_{w} \frac{\partial C_{w}}{\partial \mathbf{n}_{w}}=0 \quad \text { on } \Gamma_{w, d w} .
\end{array}\right.
$$

To complete (2.2), we add the initial conditions

$$
C_{f}(\mathbf{x}, t)=C_{f 0}(\mathbf{x}), \quad \mathbf{x} \in \Omega_{f}, \quad C_{w}(\mathbf{x}, t)=C_{w 0}(\mathbf{x}), \quad \mathbf{x} \in \Omega_{w}, \quad t=0,
$$


where the diffusivity coefficients $\mu_{f}$ and $\mu_{w}$ are positive and constant and $s_{f}(\mathbf{x}, t)$ and $s_{w}(\mathbf{x}, t)$ are possible source terms. In $(2.2)$, we have denoted by $\Gamma_{w, u p}\left(\Gamma_{w, d w}\right)$ the part of the wall corresponding to the proximal (distal) section of the fluid domain (see Figure 2.1).

In this model, observe that the solute is regarded as a passive scalar; that means that it is simply convected by the blood in the lumen, neglecting any possible feedback on the hemodynamics. In particular, viscosity and density of blood are assumed independent of the solute concentration. This hypothesis is actually coherent with the models for solute dynamics proposed in [2], [3].

Now, observe that $(2.2)_{4}$ can be equivalently substituted by the equation

$$
\mu_{f} \frac{\partial C_{f}}{\partial \mathbf{n}_{f}}=-\mu_{w} \frac{\partial C_{w}}{\partial \mathbf{n}_{w}} \quad \text { on } \Gamma
$$

prescribing the continuity of the solute flux between the fluid and the wall domains. However, we prefer the formulation (2.2), as it leads to a more efficient subdomain iterative scheme.

REMARK 2.1. For some gaseous solutes, the diffusivity coefficient $\mu_{f}$ depends on the rate of deformation of blood (see [4]). This feature makes the mathematical analysis of the coupled Navier-Stokes/solute dynamics problem more involved, as addressed in [14]. However, it does not bring significant differences in the context of the present work, which is mainly focused on the numerical approximation.

In order to carry out the mathematical analysis of the problem given by (2.1) and (2.2), as well as its numerical discretization, we resort to their "weak" or variational formulations. Concerning the Navier-Stokes equations, this can be done in a very standard way and we refer to, e.g., [12], [17]. For the advection-diffusion problem we introduce the following notation. For all $\psi_{f}, \phi_{f} \in H_{\partial \Omega_{f} \backslash \Gamma}^{1}\left(\Omega_{f}\right)$ and $\psi_{w}, \phi_{w} \in$ $H_{\partial \Omega_{w} \backslash \Gamma}^{1}\left(\Omega_{w}\right)$ set

$$
\begin{array}{r}
a_{f}\left(\psi_{f}, \phi_{f}\right)=\mu_{f}\left(\nabla \psi_{f}, \nabla \phi_{f}\right)+\left((\mathbf{u} \cdot \nabla) \psi_{f}, \phi_{f}\right), \\
a_{w}\left(\psi_{w}, \phi_{w}\right)=\mu_{w}\left(\nabla \psi_{w}, \nabla \phi_{w}\right) .
\end{array}
$$

Both $a_{f}(\cdot, \cdot)$ and $a_{w}(\cdot, \cdot)$ are continuous and coercive bilinear forms; additionally, $a_{w}(\cdot, \cdot)$ is symmetric. Then, the weak formulation of problem $(2.2)$ reads as follows.

Problem 2.1. Given the initial condition $C_{f}(\mathbf{x}, t=0)=C_{f, 0}, \in H_{\partial \Omega_{f} \backslash \Gamma}^{1}\left(\Omega_{f}\right)$ and $C_{w}(\mathbf{x}, t=0)=C_{w, 0} \in H_{\partial \Omega_{w} \backslash \Gamma}^{1}\left(\Omega_{w}\right)$ find $C_{f} \in L^{2}\left(0, T ; H_{\partial \Omega_{f} \backslash \Gamma}^{1}\left(\Omega_{f}\right)\right), C_{w} \in$ $L^{2}\left(0, T ; H_{\partial \Omega_{w} \backslash \Gamma}^{1}\left(\Omega_{w}\right)\right)$ such that for all $\phi_{f} \in H_{\partial \Omega_{f} \backslash \Gamma}^{1}\left(\Omega_{f}\right)$ and $\phi_{w} \in H_{\partial \Omega_{w} \backslash \Gamma}^{1}\left(\Omega_{w}\right)$

$$
\left\{\begin{array}{c}
\left(\frac{\partial C_{f}}{\partial t}, \phi_{f}\right)+a_{f}\left(C_{f}, \phi_{f}\right)+\left(C_{f}-C_{w}, \phi_{f}\right)_{\zeta}=\left(s_{f}, \phi_{f}\right), \\
\left(\frac{\partial C_{w}}{\partial t}, \phi_{w}\right)+a_{w}\left(C_{w}, \phi_{w}\right)+\left(C_{w}-C_{f}, \phi_{w}\right)_{\zeta}=\left(s_{w}, \phi_{w}\right) .
\end{array}\right.
$$

If the domain and the data are regular enough, Problem 2.1, coupled with the (weak form of) Navier-Stokes one, is well posed. More precisely, the following proposition can be shown (see [14]).

Proposition 2.1. If $\Omega_{f}$ is a $2 D$ domain smooth enough, then the coupled blood solute dynamics problem admits a unique solution, depending continuously on the 
data. If $\Omega_{f}$ is a $3 D$ domain, the same conclusion holds true, provided the initial data of the Navier-Stokes problem are sufficiently small.

Concerning the result of this proposition, we point out that the smallness of the data for the Navier-Stokes problem is actually a standard and an unavoidable hypothesis for ensuring the existence of a convective field in any problem involving an incompressible fluid. Indeed, no unconditional well posedness result is so far available for the Navier-Stokes equations (see [17], [12]). Moreover, observe that if the diffusivity of the solute in the lumen is the function of the shear rate (see Remark 2.1) the smallness of the initial data for the Navier-Stokes problem is necessary even for the $2 \mathrm{D}$ case.

We point out that in [14] only full Dirichlet boundary conditions for the blood were considered, which yield a coercive bilinear form. However, in the current case, a standard energy argument allows us to prove that the bilinear form is weakly coercive, which is still sufficient to ensure the well posedness of the associated parabolic problem (see [5], [6], [12]).

\section{Discretization and Steklov-Poincaré operators.}

3.1. The semidiscrete problem. We will suppose that the blood velocity and pressure are available upon solving the weak counterpart of (2.1) and focus our attention on the computation of Problem 2.1. In view of the subsequent analysis, we first introduce the semidiscrete model, namely the time-discrete counterpart of Problem 2.1. To this end, we subdivide the time interval $[0, T]$ in $N$ time steps $t^{n}=n \Delta t$, with $\Delta t>0$ and $n=1, \ldots, N$. Setting $\chi=\frac{1}{\Delta t}$, we obtain the time-discrete problem based on the backward Euler method.

Problem 3.1. Given $C_{f}^{0}$ and $C_{w}^{0}$ for every $n=0,1, \ldots, N-1$ find $C_{f}^{n+1} \in$ $H_{\partial \Omega_{f} \backslash \Gamma}^{1}\left(\Omega_{f}\right)$ and $C_{w}^{n+1} \in H_{\partial \Omega_{f} \backslash \Gamma}^{1}\left(\Omega_{w}\right)$ such that for all $\phi_{f}$ in $H_{\partial \Omega_{f} \backslash \Gamma}^{1}\left(\Omega_{f}\right)$ and $\phi_{w}$ in $H_{\partial \Omega_{w} \backslash \Gamma}^{1}\left(\Omega_{w}\right)$

$$
\left\{\begin{array}{l}
\widehat{a}_{f}\left(C_{f}^{n+1}, \phi_{f}\right)+\left(C_{f}^{n+1}, \phi_{f}\right)_{\zeta}-\left(C_{w}^{n+1}, \phi_{f}\right)_{\zeta}=\chi\left(C_{f}^{n}, \phi_{f}\right)+\left(s_{f}^{n+1}, \phi_{f}\right), \\
\widehat{a}_{w}\left(C_{w}^{n+1}, \phi_{w}\right)+\left(C_{w}^{n+1}, \phi_{w}\right)_{\zeta}-\left(C_{f}^{n+1}, \phi_{w}\right)_{\zeta}=\chi\left(C_{w}^{n}, \phi_{w}\right)+\left(s_{w}^{n+1}, \phi_{w}\right),
\end{array}\right.
$$

where $s_{f}^{n+1}=s_{f}\left(t^{n+1}\right), s_{w}^{n+1}=s_{w}\left(t^{n+1}\right), C_{f}^{0}$ and $C_{w}^{0}$ are the initial data, and

$$
\begin{gathered}
\widehat{a}_{f}\left(\psi_{f}, \phi_{f}\right)=\chi\left(\psi_{f}, \phi_{f}\right)+a_{f}\left(\psi_{f}, \phi_{f}\right) \quad \forall \phi_{f}, \psi_{f} \in H_{\partial \Omega_{f} \backslash \Gamma}^{1}\left(\Omega_{f}\right), \\
\widehat{a}_{w}\left(\psi_{w}, \phi_{w}\right)=\chi\left(\psi_{w}, \phi_{w}\right)+a_{w}\left(\psi_{w}, \phi_{w}\right) \quad \forall \phi_{w}, \psi_{w} \in H_{\partial \Omega_{w} \backslash \Gamma}^{1}\left(\Omega_{w}\right) .
\end{gathered}
$$

Observe that these bilinear forms are continuous and coercive, and, in particular, $\widehat{a}_{w}(\cdot, \cdot)$ is symmetric.

3.2. The Steklov-Poincaré interface equation. We are going to provide a different formulation of (3.1) which will be useful in view of the subsequent analysis. In what follows, the specification of the time index $n+1$ will be dropped whenever clear from the context.

Let us start with the "wall-side" of the problem. Let $\mathcal{H}_{w}: \Lambda \rightarrow H_{\partial \Omega_{w} \backslash \Gamma}^{1}\left(\Omega_{w}\right)$ be defined such that, for a given function $\rho \in \Lambda, u_{w}=\mathcal{H}_{w} \rho$ solves

$$
\left\{\begin{array}{l}
\chi u_{w}-\mu_{w} \Delta u_{w}=0 \text { in } \Omega_{w}, \\
u_{w}=\rho \text { on } \Gamma, \quad u_{w}=0 \text { on } \partial \Omega_{w} \backslash \Gamma .
\end{array}\right.
$$


Moreover, let $\mathcal{G}_{w}: L^{2}\left(\Omega_{w}\right) \rightarrow H_{0}^{1}\left(\Omega_{w}\right)$ be the operator such that, for a given function $r_{w} \in L^{2}\left(\Omega_{w}\right), g_{w}=\mathcal{G}_{w} r_{w}$ satisfies

$$
\left\{\begin{array}{l}
\chi g_{w}-\mu_{w} \Delta g_{w}=r_{w} \text { in } \Omega_{w}, \\
g_{w}=0 \text { on } \partial \Omega_{w} .
\end{array}\right.
$$

Correspondingly, we introduce the following operator $\mathcal{H}_{f}: \Lambda \rightarrow H_{\partial \Omega_{f} \backslash \Gamma}^{1}\left(\Omega_{f}\right)$ for the "fluid-side" of the problem. Given $\rho \in \Lambda, u_{f}=\mathcal{H}_{f} \rho$ satisfies

$$
\left\{\begin{array}{l}
\chi u_{f}-\mu_{f} \Delta u_{f}+\mathbf{u} \cdot \nabla u_{f}=0 \quad \text { in } \quad \Omega_{f}, \\
u_{f}=0 \quad \text { on } \partial \Omega_{f} \backslash \Gamma, \quad \mu_{f} \frac{\partial u_{f}}{\partial \mathbf{n}_{f}}+\zeta u_{f}=\zeta \rho \text { on } \Gamma .
\end{array}\right.
$$

Furthermore, $\mathcal{G}_{f}: L^{2}\left(\Omega_{f}\right) \rightarrow H_{\partial \Omega_{f} \backslash \Gamma}^{1}\left(\Omega_{f}\right)$ is such that, given $r_{f} \in L^{2}\left(\Omega_{f}\right), g_{f}=\mathcal{G}_{f} r_{f}$ solves

$$
\left\{\begin{array}{l}
\chi g_{f}-\mu_{f} \Delta g_{f}+\mathbf{u} \cdot \nabla g_{f}=r_{f} \quad \text { in } \quad \Omega_{f}, \\
g_{f}=0 \quad \text { on } \quad \partial \Omega_{f} \backslash \Gamma, \quad \mu_{f} \frac{\partial g_{f}}{\partial \mathbf{n}_{f}}+\zeta g_{f}=0 \quad \text { on } \quad \Gamma .
\end{array}\right.
$$

Finally, for a given function $\rho \in \Lambda$, we define

$$
\begin{array}{lll}
\mathcal{S}_{w}: \Lambda \rightarrow \Lambda^{\prime} & \text { such that } & \mathcal{S}_{w} \rho=\mu_{w} \frac{\partial \mathcal{H}_{w} \rho}{\partial \mathbf{n}_{w}}+\zeta \rho, \\
\mathcal{S}_{f}: \Lambda \rightarrow \Lambda^{\prime} & \text { such that } \quad \mathcal{S}_{f} \rho=-\zeta\left(\gamma_{f} \mathcal{H}_{f} \rho\right) .
\end{array}
$$

By extending a definition which is common in the framework of domain decomposition methods we call $\mathcal{S}_{f}$ and $\mathcal{S}_{w}$ the Steklov-Poincaré operators associated with the heterogeneous problem at hand. A general discussion on the role and the properties of Steklov-Poincaré (SP) operators in the framework of domain decomposition can be found in [13]. On the basis of the previous definitions, the time-discrete formulation of the problem can be reformulated in terms of an operatorial interface equation. Indeed, if we set

$$
\mathcal{S}=\mathcal{S}_{f}+\mathcal{S}_{w} \quad \text { and } \quad \eta=-\left(\mu_{f} \frac{\partial \mathcal{G}_{f} r_{f}}{\partial \mathbf{n}_{f}}+\mu_{w} \frac{\partial \mathcal{G}_{w} r_{w}}{\partial \mathbf{n}_{w}}\right),
$$

then, from $(2.2)_{4}$, the following interface equation needs to be solved for the unknown $\rho$ at each time step:

$$
\mathcal{S} \rho=\eta
$$

The SP operators can be reinterpreted in a weak from by considering (3.10) in a distributional sense. Let us denote by $\langle\cdot, \cdot\rangle$ the duality pairing of $\Lambda$ with $\Lambda^{\prime}$. By Green formula, we obtain for all $\lambda \in \Lambda$

$$
\left\langle\mathcal{S}_{w} \rho, \lambda\right\rangle=\left\langle\mu_{w} \frac{\partial \mathcal{H}_{w} \rho}{\partial \mathbf{n}_{w}}, \lambda\right\rangle+(\rho, \lambda)_{\zeta}=\widehat{a}_{w}\left(u_{w}, \mathcal{L}_{w} \lambda\right)+(\rho, \lambda)_{\zeta}
$$


with $\mathcal{L}_{w} \lambda \in H_{\partial \Omega_{w} \backslash \Gamma}^{1}\left(\Omega_{w}\right)$ and

$$
\begin{aligned}
\left\langle\mathcal{S}_{f} \rho, \lambda\right\rangle & =-\left(\mathcal{H}_{f} \rho, \lambda\right)_{\zeta}=-\left(u_{f}, \lambda\right)_{\zeta}=-(\rho, \lambda)_{\zeta}+\mu_{f}\left\langle\frac{\partial u_{f}}{\partial \mathbf{n}_{f}}, \lambda\right\rangle \\
& =-(\rho, \lambda)_{\zeta}+\widehat{a}_{f}\left(u_{f}, \mathcal{L}_{f} \lambda\right)
\end{aligned}
$$

with $\mathcal{L}_{f} \lambda \in H_{\partial \Omega_{f} \backslash \Gamma}^{1}\left(\Omega_{f}\right)$. In the latter equation, we have exploited the definition of $u_{f}$ and, in particular, $(3.6)_{2}$. Consequently,

$$
\langle\mathcal{S} \rho, \lambda\rangle=\mu_{w}\left\langle\frac{\partial \mathcal{H}_{w} \rho}{\partial \mathbf{n}_{w}}, \lambda\right\rangle+(\rho, \lambda)_{\zeta}-\left(\mathcal{H}_{f} \rho, \lambda\right)_{\zeta}=\widehat{a}_{w}\left(u_{w}, \mathcal{L}_{w} \lambda\right)+\widehat{a}_{f}\left(u_{f}, \mathcal{L}_{f} \lambda\right)
$$

for all $\lambda \in \Lambda$. By proceeding in a similar way for the right-hand side, the weak formulation of the interface relation (3.10) finally reads as follows: find $\rho \in \Lambda$ such that

$$
\widehat{a}_{w}\left(\mathcal{H}_{w} \rho+\mathcal{G}_{w} r_{w}, \mathcal{L}_{w} \lambda\right)+\widehat{a}_{f}\left(\mathcal{H}_{f} \rho+\mathcal{G}_{f} r_{f}, \mathcal{L}_{f} \lambda\right)=\left(r_{f}, \mathcal{L}_{f} \lambda\right)+\left(r_{w}, \mathcal{L}_{w} \lambda\right)
$$

for all $\lambda \in \Lambda$. Finally, we notice that, in the special case $\phi_{f}=\mathcal{L}_{f} \lambda$ and $\phi_{w}=\mathcal{L}_{w} \lambda$, and, provided that $r_{f}=\chi C_{f}^{n}+s_{f}^{n+1}, r_{w}=\chi C_{w}^{n}+s_{w}^{n+1},(3.14)$ is equivalent to (2.5).

3.3. The fully discrete problem. The space discretization of the problem is carried out using the finite element method (FEM). To this end, let us introduce $\mathcal{T}_{h f}$ and $\mathcal{T}_{h w}$, two admissible triangulations (see, e.g., [12]) of $\bar{\Omega}_{f}$ and $\bar{\Omega}_{w}$ respectively. For the sake of simplicity, we assume that $\mathcal{T}_{h f}$ and $\mathcal{T}_{h w}$ are conforming triangulations on $\Gamma$. Consequently, $\mathcal{T}_{h}=\mathcal{T}_{h f} \cup \mathcal{T}_{h w}$ is an admissible triangulation for $\bar{\Omega}_{f} \cup \bar{\Omega}_{w}$. Let $h$ be a characteristic length of the elements $K \in \mathcal{T}_{h}$ and $V_{h f}$ and $V_{h w}$ be a couple of finitedimensional subspaces of $H_{\partial \Omega_{f} \backslash \Gamma}^{1}$ and $H_{\partial \Omega_{w} \backslash \Gamma}^{1}$, respectively. Moreover, denote by $V_{h w, 0}$ a finite-dimensional subspace of $H_{0}^{1}\left(\Omega_{w}\right)$. Finally let $\Lambda_{h}$ be a finite-dimensional subspace of $\Lambda$ such that the traces on $\Gamma$ of functions in $V_{h f}$ or $V_{h w}$ belong to $\Lambda_{h}$. Denote by $N_{f}$ the dimension of $V_{h f}$, by $N_{w}$ the dimension of $V_{h w}$, and by $N_{\Gamma}$ the dimension of $\Lambda_{h}$. Let $\left\{\phi_{i, f}\right\}\left(i=1,2, \ldots, N_{f}\right)$ and similarly $\left\{\phi_{i, w}\right\}\left(i=1,2, \ldots, N_{w}\right)$ be a basis for $V_{h f}$ and for $V_{h w}$, respectively; moreover, denote with $\left\{\phi_{i, \Gamma}\right\}\left(i=1,2, \ldots, N_{\Gamma}\right)$ a basis for $\Lambda_{h}$. In what follows, the subscript $h$ will identify the space discrete solution.

Based on these definitions, the space discretization of Problem 2.1 reads as follows.

Problem 3.2. Given the initial data $C_{f h}^{0}$ and $C_{w h}^{0}$, for every $n=0,1, \ldots, N-1$ (being $N \Delta t=T$ the final time), find $C_{f h}^{n+1} \in V_{h f}$ and $C_{w h}^{n+1} \in V_{h w}$ such that for all $i=1, \ldots, N_{f}$ and $j=1, \ldots, N_{w}$

$$
\left\{\begin{array}{l}
\widehat{a}_{f}\left(C_{f h}^{n+1}, \phi_{i, f}\right)+\left(C_{f h}^{n+1}, \phi_{i, f}\right)_{\zeta}-\left(C_{w h}^{n}, \phi_{i, f}\right)_{\zeta}=\chi\left(C_{f h}^{n}, \phi_{i, f}\right)+\left(s_{f}^{n+1}, \phi_{i, f}\right), \\
\widehat{a}_{w}\left(C_{w h}^{n+1}, \phi_{j, w}\right)+\left(C_{w h}^{n+1}, \phi_{j, w}\right)_{\zeta}-\left(C_{f h}^{n+1}, \phi_{j, w}\right)_{\zeta}=\chi\left(C_{w h}^{n}, \phi_{j, w}\right)+\left(s_{w}^{n+1}, \phi_{j, w}\right) .
\end{array}\right.
$$

REMARK 3.1. Due to the convection dominated nature of the problem (see [15]), the pure Galerkin discrete formulation (3.15) does not ensure stability to the numerical solution unless the triangulation is fine enough to ensure that the local Péclet number is less than one. It is therefore mandatory to adopt suitable stabilization techniques. In particular, we will make use of a strongly consistent method like SUPG 
(streamline upwind Petrov Galerkin) - see, e.g., [12]. We address first the analysis of the scheme to the Galerkin formulation (3.15) in order to point out the intrinsic features of the method. Later on, we will address the modifications induced by the presence of stabilizing terms (see section 4.1).

3.3.1. The discrete SP operators. Let us introduce the discrete counterpart $\widehat{\mathcal{S}}_{h w}$ and $\widehat{\mathcal{S}}_{h f}$ of the SP operators. Following (3.11), we define

$$
\left\langle\widehat{\mathcal{S}}_{h w} \rho, \lambda\right\rangle=\widehat{a}_{w}\left(u_{w}, \mathcal{L}_{w} \lambda\right)+(\rho, \lambda)_{\zeta} \quad \forall \rho, \lambda \in \Lambda_{h},
$$

where

$$
u_{w} \in V_{h w}:\left\{\begin{array}{l}
\widehat{a}_{w}\left(u_{w}, \phi_{w}\right)=0 \quad \forall \phi_{w} \in V_{h w, 0} \\
u_{w}=\rho \text { on } \Gamma .
\end{array}\right.
$$

Similarly we define

$$
\left\langle\widehat{\mathcal{S}}_{h f} \rho, \lambda\right\rangle=-(\rho, \lambda)_{\zeta}+\widehat{a}_{f}\left(u_{f}, \mathcal{L}_{f} \lambda\right)
$$

where

$$
u_{f} \in V_{h f}: \widehat{a}_{f}\left(u_{f}, \phi_{f}\right)+\left(u_{f}, \phi_{f}\right)_{\zeta}=\left(\rho, \phi_{f}\right)_{\zeta} \quad \forall \phi_{f} \in V_{h f} .
$$

Finally, we set $\widehat{\mathcal{S}}_{h}=\widehat{\mathcal{S}}_{h w}+\widehat{\mathcal{S}}_{h f}$. Then, the solution of Problem 3.2 can be reformulated in terms of the interface equation

$$
\left\langle\widehat{\mathcal{S}}_{h} \rho, \lambda\right\rangle=\langle\widehat{\eta}, \lambda\rangle \quad \forall \lambda \in \Lambda,
$$

where $\rho$ stands for the trace of $C_{w h}$ on $\Gamma$, i.e.,

$$
\rho=\left.C_{w h}\right|_{\Gamma}
$$

and

$$
\widehat{\eta}=-\left(\mu_{w} \frac{\partial \mathcal{G}_{w} r_{w}}{\partial \mathbf{n}_{w}}+\mu_{f} \frac{\partial \mathcal{G}_{f} r_{f}}{\partial \mathbf{n}_{f}}\right)
$$

with

$$
r_{f}=r_{f}^{n+1}=s_{f}^{n+1}+\chi C_{f h}^{n}, \quad r_{w}=r_{w}^{n+1}=s_{w}^{n+1}+\chi C_{w h}^{n},
$$

where, for the sake of clarity, the time index has been restored.

In what follows we will use several times the so called finite element uniform extension theorem (FEUET), which states that $\left\|\rho_{h}\right\|_{\Lambda}$ is uniformly equivalent (with respect to $h)$ to $\left\|u_{h, w}\right\|_{H^{1}\left(\Omega_{w}\right)}$, where $u_{h, w}$ is the finite element approximation of problem (3.4), where $\rho$ is replaced by $\rho_{h}$; see [13, Theorem 4.1.3].

Proposition 3.1. The following properties hold for $\widehat{\mathcal{S}}_{h w}, \widehat{\mathcal{S}}_{h f}$, and $\widehat{\mathcal{S}}_{h}$ :

1. $\widehat{\mathcal{S}}_{h w}$ is continuous, symmetric, and coercive in $\Lambda_{h}$.

2. $\widehat{\mathcal{S}}_{h f}$ is continuous and negative; i.e., for any $\rho \in \Lambda_{h},\left\langle\widehat{\mathcal{S}}_{h f} \rho, \rho\right\rangle<0$.

3. $\widehat{\mathcal{S}}_{h}$ is continuous and coercive in $\Lambda_{h}$.

Proof. Continuity and symmetry of $\widehat{\mathcal{S}}_{h w}$ can be proven from the continuity and symmetry of $\widehat{a}_{w}(\cdot, \cdot)$ and the definition $(3.16)$, owing to the FEUET. Moreover, $\widehat{\mathcal{S}}_{h w}$ is coercive thanks to the coercivity of $\widehat{a}_{w}(\cdot, \cdot)$, the positivity of $\zeta$, and the trace 
inequality (1.1). Observe in particular that the continuity and coercivity constants do not depend on $h$.

Continuity of $\widehat{\mathcal{S}}_{h f}$ follows from the coercivity of the bilinear form on the righthand side of (3.18). Again, the continuity constant does not depend on $h$, depending on the bilinear form properties. Moreover, from (3.17) and taking again $\phi_{f}=u_{f}$ in (3.18), we obtain

$$
-\left\langle\widehat{\mathcal{S}}_{h f} \rho, \rho\right\rangle=\left(u_{f}, \rho\right)_{\zeta}=\widehat{a}_{f}\left(u_{f}, u_{f}\right)+\left\|u_{f}\right\|_{\zeta}^{2}>0 \quad \forall \rho \in \Lambda_{h}
$$

Item 2 is thus proven. The continuity of $\widehat{\mathcal{S}}_{h}$ is now a consequence of the continuity of $\widehat{\mathcal{S}}_{h w}$ and $\widehat{\mathcal{S}}_{h f}$. In particular, we stress that the continuity constant is independent on $h$.

Finally, we prove that $\widehat{\mathcal{S}}_{h}$ is coercive. Indeed, observe that by the definition of $u_{f}$ and (3.22)

$$
\widehat{a}_{f}\left(u_{f}, \mathcal{L}_{f} \rho\right)=\left(\rho-\gamma_{f} u_{f}, \rho\right)_{\zeta}=\left(\rho-\gamma_{f} u_{f}, \rho-\gamma_{f} u_{f}\right)_{\zeta}+\widehat{a}_{f}\left(u_{f}, u_{f}\right) .
$$

Consequently, we obtain

$$
\left\langle\widehat{\mathcal{S}}_{h} \rho, \rho\right\rangle=\widehat{a}_{w}\left(u_{w}, \mathcal{L}_{w} \rho\right)+\widehat{a}_{f}\left(u_{f}, \mathcal{L}_{f} \rho\right) \geq \widehat{a}_{w}\left(u_{w}, u_{w}\right) \geq \tau\|\rho\|_{\Lambda}^{2} .
$$

Finally, observe that the coercivity constant considered above is independent of $h$. Indeed, the coercivity constant $\tau$ in (3.24) is independent of $h$. This is due to the circumstance that $u_{w}$ is the extension of $\rho$ so that we can advocate once again the FEUET.

As a direct consequence of the previous properties, we obtain the following corollary.

COROLlary 3.1. The interface equation (3.19) admits a unique solution which depends continuously on the data.

Proof. The functional $\mathcal{F}(\lambda) \equiv\langle\widehat{\eta}, \lambda\rangle$ associated with the right-hand side of (3.19) is continuous in $\Lambda_{h}$. Moreover, the bilinear form $\left\langle\widehat{\mathcal{S}}_{h} \cdot, \cdot\right\rangle$ is continuous and coercive in $\Lambda_{h}$, as proved in Proposition 3.1. The result then follows from the Lax-Milgram lemma.

4. The subdomain iterative method. In order to reduce the computational cost required by the numerical solution of Problem 3.2, we suitably split the whole problem into a sequence of subproblems to be solved in the two physical subdomains (the lumen $\Omega_{f}$ and the wall $\Omega_{w}$ ). In [14] we have introduced and analyzed an iterative scheme based on the interface conditions $(2.2)_{3}$ and $(2.2)_{4}$. In the present work, we consider a relaxed extension of the scheme in order to speed up the convergence. We will extend the convergence results proven in [14] to the relaxed algorithm, taking advantage of the reinterpretation of the scheme in terms of SP operators. (That was not considered at all in [14].) In what follows, for notational convenience we will drop the specification $h$, for the space discrete quantities, as well as the specification $n+1$ for the time-discrete quantities (i.e., we will write $\left[C_{f, k}, C_{w, k}\right]$ instead of $\left[C_{f h, k}^{n+1}, C_{w h, k}^{n+1}\right]$ ). It is understood, however, that the iterative method is carried out on the fully discrete problem.

The scheme which we adopt is the following. Given an initial guess $\rho_{0}$ for $C_{w, 0}$ on the interface $\Gamma$, for $k=0,1, \ldots$ find the sequence of functions $\left[C_{f, k}, C_{w, k}\right] \in V_{h f} \times V_{h w}$ by solving for all $i=1, \ldots, N_{f}$ and $j=1, \ldots, N_{w}$

$$
\widehat{a}_{f}\left(C_{f, k+1}, \phi_{i, f}\right)+\left(C_{f, k+1}, \phi_{i, f}\right)_{\zeta}=\left(r_{f}, \phi_{i, f}\right)+\left(\rho_{k}, \phi_{i, f}\right)_{\zeta},
$$




$$
\begin{gathered}
\widehat{a}_{w}\left(C_{w, k+1}, \phi_{j, w}\right)+\left(C_{w, k+1}, \phi_{j, w}\right)_{\zeta}=\left(r_{w}, \phi_{j, w}\right)+\left(C_{f, k+1}, \phi_{j, w}\right)_{\zeta} \\
\rho_{k+1}=\left.C_{w, k+1}\right|_{\Gamma}
\end{gathered}
$$

where $r_{f}$ and $r_{w}$ are defined in (3.21).

This is a Robin-Robin iterative scheme, as it is based on the conditions $(2.2)_{3}$ and $(2.2)_{4}$, which are Robin conditions for each subproblem involved.

In order to resort to an iterative scheme for the variable $\rho_{k+1}$ alone, let us eliminate the unknowns $C_{f, k+1}, C_{w, k+1}$ from (4.1)-(4.3).

Let us take $\phi_{f}=\mathcal{L}_{f} \lambda$ in (4.1), where $\lambda$ is any function of $\Lambda_{h}$, and split $C_{f, k+1}$ as $C_{f, k+1}=u_{f, k+1}+g_{f}$, where $u_{f, k+1}$ and $g_{f}$ solve, respectively, problems (3.6) and (3.7) in a weak sense. Recalling (3.17), we have

$$
\begin{aligned}
& \left(C_{f, k+1}, \lambda\right)_{\zeta}=\left(r_{f}, \mathcal{L}_{f} \lambda\right)+\left(\rho_{k}, \lambda\right)_{\zeta}-\widehat{a}_{f}\left(u_{f, k+1}, \mathcal{L}_{f} \lambda\right)-\widehat{a}_{f}\left(g_{f}, \mathcal{L}_{f} \lambda\right) \\
& =-\left\langle\nabla g_{f} \cdot \mathbf{n}_{f}, \lambda\right\rangle-\left\langle\widehat{\mathcal{S}}_{h f} \rho_{k}, \lambda\right\rangle \quad \forall \lambda \in \Lambda_{h} .
\end{aligned}
$$

Proceeding similarly on (4.2) and recalling that $C_{w, k+1}=u_{w, k+1}+g_{w}$, we obtain

$$
\left\langle\widehat{\mathcal{S}}_{h w} C_{w, k+1}, \lambda\right\rangle=\widehat{a}_{w}\left(u_{w, k+1}, \mathcal{L}_{w} \lambda\right)+\left(C_{w, k+1}, \lambda\right)_{\zeta} \quad \forall \lambda \in \Lambda_{h}
$$

and

$$
\widehat{a}_{w}\left(g_{w}, \mathcal{L}_{w} \lambda\right)=\left(r_{w}, \mathcal{L}_{w} \lambda\right)+\left\langle\nabla g_{w} \cdot \mathbf{n}_{w}, \lambda\right\rangle \quad \forall \lambda \in \Lambda_{h} .
$$

Consequently, taking $\phi_{w}=\mathcal{L}_{w} \lambda$ in (4.2), by virtue of the latter two equations we have

$$
\left\langle\widehat{\mathcal{S}}_{h w} C_{w, k+1}, \lambda\right\rangle+\left\langle\nabla g_{w} \cdot \mathbf{n}_{w}, \lambda\right\rangle=\left(C_{f, k+1}, \lambda\right)_{\zeta} \quad \forall \lambda \in \Lambda_{h} .
$$

Substituting (4.4) in the latter, we obtain

$$
\left\langle\widehat{\mathcal{S}}_{h w} C_{w, k+1}, \lambda\right\rangle=\left\langle\widehat{\mathcal{S}}_{h w} \rho_{k+1}, \lambda\right\rangle=\langle\widehat{\eta}, \lambda\rangle-\left\langle\widehat{\mathcal{S}}_{h f} \rho_{k}, \lambda\right\rangle \quad \forall \lambda \in \Lambda_{h},
$$

where from (3.20)

$$
\widehat{\eta}=-\left(\mu_{f} \nabla g_{f} \cdot \mathbf{n}_{f}+\mu_{w} \nabla g_{w} \cdot \mathbf{n}_{w}\right) .
$$

If we consider a relaxation parameter $\theta,(4.3)$ becomes

$$
\rho_{k+1}=\left.\theta C_{w, k+1}\right|_{\Gamma}+(1-\theta) \rho_{k} .
$$

Hence for all $\lambda \in \Lambda_{h}$, by means of (4.5), we obtain

$$
\left\langle\widehat{\mathcal{S}}_{h w}\left(\rho_{k+1}-\rho_{k}\right), \lambda\right\rangle=\theta\left\langle\widehat{\mathcal{S}}_{h w} C_{w, k+1}, \lambda\right\rangle-\theta\left\langle\widehat{\mathcal{S}}_{h w} \rho_{k}, \lambda\right\rangle=\theta\left\langle\widehat{\eta}-\widehat{\mathcal{S}}_{h} \rho_{k}, \lambda\right\rangle .
$$

Equation (4.7) actually sheds light on a useful reinterpretation of the Robin-Robin method, which can be regarded as a preconditioned Richardson method for problem (3.19), with $\widehat{\mathcal{S}}_{h w}$ playing the role of preconditioner for $\widehat{\mathcal{S}}_{h}$. This observation allows for a straightforward proof of convergence of the scheme. Indeed, we have the following abstract result (for the proof, see, e.g., [13, Theorem 4.2.2 and Remark 4.2.4]).

Theorem 4.1. Let $X$ be a real Hilbert space, $X^{\prime}$ its dual space, and $\langle\cdot, \cdot\rangle$ the duality paring between $X^{\prime}$ and $X$. Let $\mathcal{Q}: X \rightarrow X^{\prime}$ be a linear invertible continuous 
operator which can be split as $\mathcal{Q}=\mathcal{Q}_{1}+\mathcal{Q}_{2}$, where $\mathcal{Q}_{i}(i=1,2)$ are linear operators. Consider the problem

$$
\mathcal{Q} \rho=\eta,
$$

where $\eta \in X^{\prime}$ is given and $\rho \in X$ is to be determined. Suppose that $\mathcal{Q}_{2}$ is continuous, symmetric, and coercive and that $\mathcal{Q}$ is coercive. Then, there exists a real value $\theta_{\text {max }}$, depending on the continuity and the coercivity constants, such that for any $\theta \in\left(0, \theta_{\max }\right)$, the sequence

$$
\rho_{k+1}=\rho_{k}+\theta \mathcal{Q}_{2}^{-1}\left(\eta-\mathcal{Q} \rho_{k}\right)
$$

converges in $X$ to the solution of (4.8) for any $\rho_{0} \in X$.

We point out that (4.9) is the generic iteration of a preconditioned Richardson method to solve (4.8), with $\mathcal{Q}_{2}$ acting as preconditioner (see [16], [9]).

Now, if we set $X=\Lambda_{h}, \mathcal{Q}=\widehat{\mathcal{S}}_{h}, \mathcal{Q}_{1}=\widehat{\mathcal{S}}_{h f}, \mathcal{Q}_{2}=\widehat{\mathcal{S}}_{h w}$, we apply this result to prove the convergence of the sequence $\left\{\rho_{k}\right\}, k \geq 0$ to the solution of (3.19). Indeed, all the hypotheses of the theorem are verified, as proven in the previous section.

Observe, moreover, that, in Theorem 4.1, the convergence rate as well as the optimal value for $\theta$ are functions of the coercivity and continuity constants of $\mathcal{Q}_{2}$ and of $\mathcal{Q}$ that in our case read $\widehat{\mathcal{S}}_{h w}$ and $\widehat{\mathcal{S}}_{h w}$, respectively. In proving Proposition 3.1 we pointed out that these constants are independent of the mesh size $h$ in our problem. This means that the rate of convergence of the proposed preconditioned iterations is not affected by the mesh size, or, in other words, that the preconditioner is optimal.

Altogether, these observations can be collected in the following final result.

Proposition 4.1. The Robin-Robin iterative scheme (4.1), (4.2), and (4.6) is convergent for any $\theta \in\left(0, \theta_{\max }\right)$, and its rate of convergence is independent of the spatial discretization. Precisely, there exists $K<1$ such that for any $\theta \in\left(0, \theta_{\max }\right)$, there exists a constant $K_{\theta} \leq K$ such that

$$
\left\|\rho-\rho_{k+1}\right\|_{\Lambda} \leq K_{\theta}\left\|\rho-\rho_{k}\right\|_{\Lambda}, \quad k \geq 0 .
$$

In agreement with this conclusion, Table 4.1 shows that the convergence rate of the relaxed Robin-Robin scheme is uniformly independent of the parameter $h$ (which in our computations with uniform grids is related to the number $N$ of finite elements nodes through the law $N \approx \mathcal{O}\left(h^{-2}\right)$ ).

REMARK 4.1. The convergence result of Theorem 4.1 refers to the preconditioned Richardson iterative method. However, similar conclusions can be shown when more efficient methods are applied to the same problem (see Table 4.1), such as the generalized minimal residual (GMRES) (see [16]), as proven in a very abstract form in $[13$, sect. 4.2.1]. On the basis of this result, we will proceed with the GMRES methods later on in section 4.3 .

4.1. The iterative method in the convection dominated case. As previously pointed out, when advection dominated problems are considered, stabilization techniques are required. This means that the bilinear form $\widehat{a}_{f}(\cdot, \cdot)$ introduced in (3.2) is substituted by

$$
\widehat{a}_{f, s t a b}\left(C_{f}, \phi_{f}\right)=\widehat{a}_{f}\left(C_{f}, \phi_{f}\right)+a_{f, h}\left(C_{f}, \phi_{f}\right),
$$

where $a_{f, h}\left(C_{f}, \phi_{f}\right)$ depends on the specific stabilization method. For instance (see, e.g., [12]), if we set

$$
L_{s} C=-\nabla \cdot \mu_{f} \nabla C, \quad L_{s s} C=\frac{1}{2} \nabla \cdot \mathbf{u} C+\frac{1}{2} \mathbf{u} \cdot \nabla C \quad\left(L_{f}=L_{s}+L_{s s}\right),
$$


TABLE 4.1

Comparison of the number of iterations to reach convergence. In all these tests we have taken $\Omega_{f}=(0,4) \times(0,1), \Omega_{w}=(0,4) \times(-1,0), u_{x}=4 u_{0}(1-y) y, u_{y}=0$. (A) $\mu_{f}=\mu_{w}=1.0 \mathrm{~cm}^{2} s^{-1}$, and $\zeta=1.0 \mathrm{~cm} \mathrm{~s}^{-1}$. (Values ${ }^{*}$ refer to the pure Galerkin method, the other to the stabilized SUPG method.) (B) $\mu_{f}=\mu_{w}=10^{-3} \mathrm{~cm}^{2} \mathrm{~s}^{-1}$ and $\zeta=1.0 \mathrm{~cm} \mathrm{~s}^{-1}$. Finer grids are obtained by means of a uniform refinement plus regularization; thus $N=\mathcal{O}\left(h^{-2}\right)$. For large values of $\zeta$ the coupled problem is severely ill-conditioned, yet the number of iterations is uniformly independent of $h$.

\begin{tabular}{|c|c|c|c|c|}
\hline$(\mathrm{A})$ & & & & \\
\hline $\mathrm{h}$ & $\mathrm{N}$ & Unrelax. Rich. & Relax. Rich. & P-GMRES \\
\hline 0.1 & 4000 & $4^{*}$ & $4^{*}$ & $3^{*}$ \\
\hline 0.05 & 16000 & $4^{*}$ & $4^{*}$ & $3^{*}$ \\
\hline 0.025 & 60000 & $4^{*}$ & $4^{*}$ & $3^{*}$ \\
\hline 0.01875 & 106000 & $4^{*}$ & $4^{*}$ & $3^{*}$ \\
\hline 0.012 & 260000 & $4^{*}$ & $4^{*}$ & $3^{*}$ \\
\hline \hline$(\mathrm{B})$ & & & & \\
\hline $\mathrm{h}$ & $\mathrm{N}$ & Unrelax. Rich. & Relax. Rich. & P-GMRES \\
\hline 0.1 & 4000 & 8 & 7 & 5 \\
\hline 0.05 & 16000 & 12 & 10 & 6 \\
\hline 0.025 & 60000 & 20 & 15 & 7 \\
\hline 0.01875 & 106000 & 23 & 17 & 7 \\
\hline 0.012 & 260000 & $29-29^{*}$ & $20-20^{*}$ & $8-8^{*}$ \\
\hline
\end{tabular}

the symmetric and the skew-symmetric parts of the fluid differential operator $L_{f}$, respectively, then the most common strongly consistent stabilization methods resort to set

$$
a_{f, h}\left(C_{f}, \phi_{f}\right)=\sum_{K \in \mathcal{T}_{h}} \delta\left(L C_{f}, \frac{h_{K}}{|\mathbf{u}|}\left(L_{s s}+\kappa L_{s}\right) \phi_{f}\right)_{K},
$$

where $K$ is the generic element of the triangulation $\mathcal{T}_{h}$ (supposed to be regular) with diameter $h_{K},(\cdot, \cdot)_{K}$ denotes the $L^{2}(K)$ scalar product, $\delta$ is a parameter to be chosen, and $\kappa$ identifies the different stabilization techniques. In particular, SUPG corresponds to set $\kappa=0$, while the Galerkin least squares (GaLS) method is given for $\kappa=1$. The Douglas-Wang (DW) method, on the other hand, corresponds to $\kappa=-1$. For the SUPG method, we recall that if $\delta$ is suitably chosen, it is possible to prove that the stabilized bilinear form $\widehat{a}_{f, s t a b}(\cdot, \cdot)$ is coercive, the constant of coercivity being independent of $h$ (see [12, Proposition 8.4.1]). A similar result holds for the DW and for the GaLS methods. In the latter case, the coercivity holds for any positive $\delta$.

Starting form these results, we are able to prove that the convergence rate of the iterative subdomains method proposed is independent of $h$ even in the stabilized case. First of all, the problem we consider in this case, in terms of SP operators can be formulated in a way completely similar to the one proposed in the previous section, provided that $\widehat{\mathcal{S}}_{h f}$ defined in (3.17) and (3.18) is substituted by

$$
\left\langle\widehat{\mathcal{S}}_{h f, s t a b} \rho, \lambda\right\rangle=-(\rho, \lambda)_{\zeta}+\widehat{a}_{f, s t a b}\left(u_{f}, \mathcal{L}_{f} \lambda\right),
$$

with

$$
u_{f} \in V_{h f}: \widehat{a}_{f, s t a b}\left(u_{f}, \phi_{f}\right)+\left(u_{f}, \phi_{f}\right)_{\zeta}=\left(\rho, \phi_{f}\right)_{\zeta} \quad \forall \phi_{f} \in V_{h f} .
$$

The crucial point is to prove that the stabilized SP operator $\widehat{\mathcal{S}}_{h f, h}$ is also continuous with a continuity constant independent of $h$. Actually, observe that by definition

$$
\left\langle\widehat{\mathcal{S}}_{h f, s t a b} \rho, \lambda\right\rangle=\left(u_{f}, \lambda\right)_{\zeta} .
$$


From (4.12) for $\phi_{f}=u_{f}$, we deduce that

$$
\left\|\gamma_{f} u_{f}\right\|_{\Lambda} \leq \beta\|\rho\|_{\Lambda}
$$

where $\beta$ is a constant obtained as a function of the trace and coercivity constants and therefore independent of $h$. The continuity constant of $\widehat{\mathcal{S}}_{h f, s t a b}$ is thus independent of $h$. Consequently, the extension of Proposition 3.1 to the stabilized case is straightforward. We therefore conclude that Theorem 4.1 can be applied as well in the stabilized case, again having independence of the convergence rate and the optimal value of $\theta$ on $h$.

4.2. Algebraic reinterpretation of the iterative scheme. The coupled Problem 3.2 requires at each time step the solution of a system in the form

$$
A \mathbf{c}=\mathbf{b} .
$$

Denoted by $N_{\Gamma}$ the degrees of freedom associated with the interface $\Gamma$, the number of degrees of freedom associated with the inner nodes in $\Omega_{w}$ is given by $N_{w 0}=N_{w}-$ $N_{\Gamma}$. Consequently, in (4.13), $\mathbf{c}=\left[\mathbf{c}_{f}, \mathbf{c}_{w}, \mathbf{c}_{\Gamma}\right]^{T} \in \mathbb{R}^{N_{f}+N_{w}}$ is the vector of unknowns specifying the discrete solution, with $\mathbf{c}_{f} \in \mathbb{R}^{N_{f}}, \quad \mathbf{c}_{w} \in \mathbb{R}^{N_{w 0}}, \quad \mathbf{c}_{\Gamma} \in \mathbb{R}^{N_{\Gamma}} . \mathbf{b} \in$ $\mathbb{R}^{N_{f}+N_{w}}$ is a function of the forcing terms, the boundary conditions, and the solution computed at the previous steps and can be correspondingly split as $\mathbf{b}=\left[\mathbf{b}_{f}, \mathbf{b}_{w}, \mathbf{b}_{\Gamma}\right]^{T}$, $\mathbf{b}_{f} \in \mathbb{R}^{N_{f}}, \quad \mathbf{b}_{w} \in \mathbb{R}^{N_{w 0}}, \quad \mathbf{b}_{\Gamma} \in \mathbb{R}^{N_{\Gamma}}$. Finally, $A \in \mathbb{R}^{\left(N_{f}+N_{w}\right) \times\left(N_{f}+N_{w}\right)}$ has the following blockwise pattern:

$$
A=\left[\begin{array}{ccc}
A_{f f} & \mathbf{0} & A_{f \Gamma} \\
\mathbf{0} & A_{w w} & A_{w \Gamma} \\
A_{\Gamma f} & A_{\Gamma w} & A_{\Gamma \Gamma}
\end{array}\right],
$$

where $A_{f f}$ is the $N_{f} \times N_{f}$ matrix associated with the discretization of the bilinear form $\widehat{a}_{f}\left(\psi_{f}, \phi_{f}\right)+\left(\psi_{f}, \phi_{f}\right)_{\zeta}$ for $\psi_{f}, \phi_{f} \in V_{h f}$. Correspondingly, $A_{f \Gamma}$ arises from the discretization of the term $-\left(\lambda, \phi_{f}\right)_{\zeta}$ (with $\lambda \in \Lambda_{h}$ ), while $A_{w w}$ is associated with the discretization of $\widehat{a}_{w}\left(\psi_{w 0}, \phi_{w 0}\right)$ for $\psi_{w 0}, \phi_{w 0} \in V_{h w, 0}$ (functions with null trace on $\Gamma)$ and $A_{w \Gamma}$ refers to the discretization of $\widehat{a}_{w}\left(\mathcal{L}_{w} \lambda, \phi_{w 0}\right)$ for $\lambda \in \Lambda_{h} . A_{\Gamma \Gamma}$ is related to the discretization of $\widehat{a}_{w}\left(\mathcal{L}_{w} \lambda, \mathcal{L}_{w} \rho\right)+(\lambda, \rho)_{\zeta}$ for $\lambda, \rho \in \Lambda_{h}$. Finally, we note that $A_{\Gamma f}=A_{f \Gamma}^{T}$ and $A_{\Gamma w}=A_{w \Gamma}^{T}$ and $A_{w w}$ and $A_{\Gamma \Gamma}$ are symmetric.

The algebraic reinterpretation of the Robin-Robin iterative scheme readily follows. Our substructuring iterative method resorts to a preconditioned Richardson scheme for (4.13),

$$
Q\left(\mathbf{c}_{k+1}-\mathbf{c}_{k}\right)=\theta\left(\mathbf{b}-A \mathbf{c}_{k}\right)=\theta \mathbf{r}_{k}, \quad k \geq 0,
$$

in which the matrix

$$
Q=\left[\begin{array}{ccc}
A_{f f} & 0 & 0 \\
0 & A_{w w} & A_{w \Gamma} \\
A_{\Gamma f} & A_{\Gamma w} & A_{\Gamma Г}
\end{array}\right]
$$

is the preconditioner. 
If we formally eliminate $\mathbf{c}_{f}$ and $\mathbf{c}_{w}$ in (4.13), we obtain the reduced system

$$
\Sigma \mathbf{c}_{\Gamma}=\boldsymbol{\eta}
$$

with $\boldsymbol{\eta}=\mathbf{b}_{\Gamma}-A_{\Gamma f} A_{f f}^{-1} \mathbf{b}_{f}-A_{\Gamma w} A_{w w}^{-1} \mathbf{b}_{w}$ and $\Sigma=A_{\Gamma \Gamma}-A_{\Gamma w} A_{w w}^{-1} A_{w \Gamma}-A_{\Gamma f} A_{f f}^{-1} A_{f \Gamma}$. Consequently, we obtain the splitting $\Sigma=\Sigma_{w}+\Sigma_{f}$, where $\Sigma_{w}=A_{\Gamma \Gamma}-A_{\Gamma w} A_{w w}^{-1} A_{w \Gamma}$, $\Sigma_{f}=A_{\Gamma f} A_{f f}^{-1} A_{f \Gamma}$. Matrix $\Sigma$ is the Schur complement of matrix A. Equation (4.17) represents the finite-dimensional counterpart of (3.19), and $\Sigma$ is the algebraic counterpart of the SP operator $\widehat{\mathcal{S}}_{h}$, while $\Sigma_{f}$ and $\Sigma_{w}$ play the role of $\widehat{\mathcal{S}}_{h f}$ and $\widehat{\mathcal{S}}_{h w}$, respectively.

By means of the block $L U$ factorization of $A$, we can explicitly compute the iteration matrix associated with the following iterative scheme:

$$
I-\theta Q^{-1} A=\left[\begin{array}{ccc}
(1-\theta) I_{f} & 0 & -\theta A_{f f}^{-1} A_{f \Gamma} \\
0 & (1-\theta) I_{w} & -\theta A_{w w}^{-1} A_{w \Gamma} \Sigma_{w}^{-1} A_{\Gamma f} A_{f f}^{-1} A_{f \Gamma} \\
0 & 0 & I-\theta \Sigma_{w}^{-1} \Sigma
\end{array}\right] .
$$

In particular, on the third block the system (4.15) yields

$$
\Sigma_{w}\left(\mathbf{c}_{\Gamma, k+1}-\mathbf{c}_{\Gamma, k}\right)=\theta\left(\boldsymbol{\eta}-\Sigma \mathbf{c}_{\Gamma, k}\right) .
$$

The latter relation enlightens the role of the matrix $\Sigma_{w}$ as a preconditioner for the Schur complement in the interface problem (4.17). As the algebraic counterpart of the operator $\widehat{\mathcal{S}}_{h w}, \Sigma_{w}$ is symmetric and positive definite.

From this perspective, we can reformulate Proposition 4.1 in the following manner.

Proposition 4.2. The preconditioned Richardson scheme (4.15) converges for any $\theta$ belonging to a suitable interval $\left(0, \theta_{\max }\right)$. The preconditioner $Q$ given in (4.16) is optimal, making the rate of convergence independent of the space discretization.

4.3. Acceleration strategies. The reinterpretation of the Robin-Robin scheme based on the Richardson framework has the advantage of highlighting that $\Sigma_{w}$ is an optimal preconditioner for $\Sigma$. (As well, $Q$ is an optimal preconditioner for $A$.) On this ground, we will take advantage of these preconditioners when more efficient iterative procedures will be applied. We will start analyzing the effects of the static relaxation parameter in the Richardson framework, and then we will consider dynamical strategies such as GMRES (see [16]).

Stationary Richardson methods. In order to compare the unrelaxed and the relaxed schemes, observe that the behavior of the iteration matrix $I-\theta Q^{-1} A$ is governed by the third diagonal block $I-\theta \Sigma_{w}^{-1} \Sigma$. In our case, $\Sigma_{w}$ and $\Sigma$ are positive definite, but $\Sigma$ is not symmetric since $A_{f f}$ is not symmetric (due to the convection term). Therefore we cannot assert a priori that $\Sigma_{w}^{-1} \Sigma$ has real positive eigenvalues; thus, an optimal static choice of $\theta$ is not straightforward (see, e.g., [9]). However, as a heuristic choice, parameter $\theta$ is selected assuming that $\Sigma$ is symmetric, setting

$$
\theta=\frac{2}{\lambda_{\min }+\lambda_{\max }},
$$

where $\sigma=\left\{\lambda_{i}\right\} i=1, \ldots, N_{\Gamma}$ is the spectrum of $\Sigma_{w}^{-1} \Sigma$, for which a rough estimate can be obtained as follows. We observe that since $I-\Sigma_{w}^{-1} \Sigma=-\Sigma_{w}^{-1} \Sigma_{f}$, the iteration matrix associated with (4.18), in the unrelaxed case, and since $\tau=\left\{\mu_{i}\right\}$ (for $i=$ $\left.1, \ldots, N_{\Gamma}\right)$ is its spectrum, the following inequalities hold:

$$
\lambda_{i}=1-\mu_{i} \text { and } \mu_{i} \leq\left\|-\Sigma_{w}^{-1} \Sigma_{f}\right\|_{p} \quad \forall i=1, \ldots, N_{\Gamma} \quad \forall p>0,
$$




$$
M_{p}:=\frac{\left\|\mathbf{c}_{\Gamma}^{k}-\mathbf{c}_{\Gamma}^{k-1}\right\|_{p}}{\left\|\mathbf{c}_{\Gamma}^{k-1}-\mathbf{c}_{\Gamma}^{k-2}\right\|_{p}} \leq\left\|-\Sigma_{w}^{-1} \Sigma_{f}\right\|_{p} .
$$

Consequently, making the approximation, $M_{p} \simeq\left\|-\Sigma_{w}^{-1} \Sigma_{f}\right\|_{p}$, and noticing that $\lambda_{\min }+\lambda_{\max }=2-\mu_{\max }-\mu_{\min }$ we make the following choice: $\theta \simeq \frac{2}{2-M_{p}}$.

Table 4.1 resumes the comparison of these methods for a diffusion dominated case (A) and an advection dominated one (B). From these results we see that, when the global matrix $A$ is ill-conditioned, the relaxation technique enhances the convergence performances with respect to the unrelaxed case.

Dynamical choice of $\theta$. In this approach the choice of $\theta$ is pursued automatically by the chosen algorithm.

Recalling that the matrix $A$ is positive definite but not symmetric, we consider, for instance, the preconditioned generalized minimal residual (P-GMRES) method (see, for example, [12], [16]), where $Q$ is the preconditioner for the global system $A \mathbf{c}=\mathbf{b}$. As we have already pointed out in Remark 4.1, $Q$ being the optimal preconditioner derived from the previous analysis, the convergence rate of the P-GMRES algorithm is independent of the number of degrees of freedom of the global system $A \mathbf{c}=\mathbf{b}$. Table 4.1 shows that the P-GMRES method performs better than the stationary strategies for both test cases.

Finally, we point out that for these tests the coefficients $\mu_{f}, \mu_{w}, \zeta$ have been chosen with the purpose of making the matrix $A$ very ill-conditioned; this explains the different performance of the considered iterative methods. However, when $\mu_{f}$, $\mu_{w}, \zeta$ are chosen in the biological range for the specific application at hand, the condition number of $A$ is lower; thus the number of iterations necessary to reach convergence is smaller for each one of the considered methods. On the other hand, for the bioengineering applications, a very large number of unknowns is required; consequently, most of the computational time is spent for solving the subsystems $Q \mathbf{z}=\mathbf{r}$ deriving from the preconditioning step. This explains why we have based the choice of an efficient iterative method not only on the number of iterations but also on the computational cost of each single iteration. In particular, a reduction of the time necessary to pursue each iteration is, for example, obtained by resorting to flexible preconditioned iterative solvers (see [16]). Among all the iterative methods considered above, the only one that allows a flexible variant is the P-GMRES. Let us briefly introduce it.

In the framework of descent methods, the computation of the descent direction $\mathbf{z}$ is carried out by solving a system $Q \mathbf{z}=\mathbf{r}$ ( $\mathbf{r}$ being the residual) which in our case, from definition (4.16), requires the solution of two subsystems:

$$
\begin{gathered}
A_{f f} \mathbf{z}_{f}=\mathbf{r}_{f}, \\
{\left[\begin{array}{cc}
A_{w w} & A_{w \Gamma} \\
A_{\Gamma w} & A_{\Gamma \Gamma}
\end{array}\right]\left[\begin{array}{c}
\mathbf{z}_{w} \\
\mathbf{z}_{\Gamma}
\end{array}\right]=\left[\begin{array}{c}
\mathbf{r}_{w} \\
\mathbf{r}_{\Gamma}
\end{array}\right]-\left[\begin{array}{c}
0 \\
A_{\Gamma f} \mathbf{r}_{f}
\end{array}\right],}
\end{gathered}
$$

which can be carried out by means of iterative methods such as BiCGStab or GMRES. The end of this flexible strategy is to compute the global solution $A \mathbf{c}=\mathbf{b}$ by solving (4.21) and (4.22) with a large tolerance, reducing henceforth the computational cost of the solution process. This can be done through the algorithm described below. Let us define with $\hat{Q}_{j}^{-1} j=1,2,3, \ldots$ an approximation of $Q^{-1}$ (obtained in our case by a low cost solution of (4.21), (4.22)) then the flexible P-GMRES (F-P-GMRES) algorithm reads (see [16]) as follows. 
TABLE 4.2

Quantitative comparison between P-GMRES and F-P-GMRES for different test cases. This example shows that the F-P-GMRES method and the proposed choice of $q$ work well for different geometries, discretized with different triangulations sharing, however, a comparable number of nodes. Note that the tolerance of the outer iterative procedure is always the same (equal to $10^{-10}$ ).

\begin{tabular}{|c|c|c|}
\hline & $\begin{array}{c}\text { P-GMRES } \\
(p=10)\end{array}$ & $\begin{array}{c}\text { F-P-GMRES } \\
(p=10, q=5)\end{array}$ \\
\hline Rectangular domains ( $h=0.012)$ & \multicolumn{2}{|c|}{} \\
\hline Iterations & 2 & 2 \\
\hline Time/iteration (s) (aver.) & 239.18 & 115.51 \\
\hline Carotid bifurcation & \multicolumn{2}{|}{} \\
\hline Iterations & 3 & 3 \\
\hline Time/iteration (s) (aver.) & 35.93 & 15.96 \\
\hline Stenosed artery & \multicolumn{2}{|}{} \\
\hline Iterations & 3 & 3 \\
\hline Time/iteration (s) (aver.) & 92.43 & 40.04 \\
\hline
\end{tabular}

Algorithm 4.1. F-P-GMRES.

Compute $\mathbf{z}_{0}=\hat{Q}_{0}^{-1}\left(\mathbf{b}-A \mathbf{x}_{0}\right), \beta=\left\|\mathbf{z}_{0}\right\|_{2}, \mathbf{v}_{1}=\mathbf{r}_{0} / \beta$.

For $j=1, \ldots, m$ Do:

compute $\mathbf{w}=A \mathbf{v}_{j}$,

compute $\mathbf{z}_{j}=\hat{Q}_{j}^{-1} \mathbf{w}$.

For $i=1, \ldots, j$ Do:

$h_{i, j}=\left(\mathbf{w}, \mathbf{v}_{i}\right)$,

$\mathbf{w}=\mathbf{w}-h_{i, j} \mathbf{v}_{i}$,

End Do.

Compute $h_{j+1, j}=\|\mathbf{w}\|_{2}$ and $\mathbf{v}_{j+1}=\mathbf{w} / h_{j+1, j}$.

End Do.

Define $Z_{m}=\left[\mathbf{z}_{1}, \ldots, \mathbf{z}_{m}\right], H_{m}=\left\{h_{i, j}\right\}_{1 \leq i \leq j+1 ; 1 \leq j \leq m}$.

Compute $\mathbf{y}_{m}=\operatorname{argmin}_{y}\left\|\beta \mathbf{e}_{1}-H_{m} \mathbf{y}\right\|_{2}$, and $\mathbf{x}_{m}=\mathbf{x}_{0}+Z_{m} \mathbf{y}_{m}$.

If $\left\|\hat{Q}_{m}^{-1}\left(\mathbf{b}-A \mathbf{x}_{m}\right)\right\|_{2}<$ tol $=10^{-p} p \in N$ then end, else $\mathbf{x}_{0} \leftarrow \mathbf{x}_{m}$.

A possible strategy to choose $\hat{Q}_{j}^{-1}$ at each iteration is as follows. Should $10^{-p}$ be the tolerance fixed in Algorithm 4.1, the tolerance of the iterative method applied to solve systems (4.21), (4.22) is set to $10^{-q}$, with $q=[p / 2]$.

For the application at hand, by adopting this strategy one obtains that F-PGMRES requires less CPU time to converge than P-GMRES (see Table 4.2).

As shown in Table 4.2, the application of the F-P-GMRES method (always based on the preconditioner provided by $\widehat{\mathcal{S}}_{h w}$ ) is very efficient. Comparing it with GMRES we see in fact that, although we have reduced the tolerance of the preconditioning step, consequently reducing the efficiency of the preconditioner, the number of global iterations does not increase. Thus the reduction in the computational cost at each iteration directly provides a reduction in the time needed to solve $A \mathbf{c}=\mathbf{b}$. Moreover, Table 4.2 shows that the considered algorithms behave well in the case of different geometries. In particular, we have considered test cases that are relevant for hemodynamics, the stenosed artery, and the carotid bifurcation.

5. Numerical results in case of physiological interest. In the present section we show some numerical results about blood and oxygen dynamics in the carotid bifurcation, a site of relevant interest in biomedical applications. We point out that our main concern is to test the efficiency of the proposed iterative method and not to give significant results from a quantitative point of view. For this reason, the model 

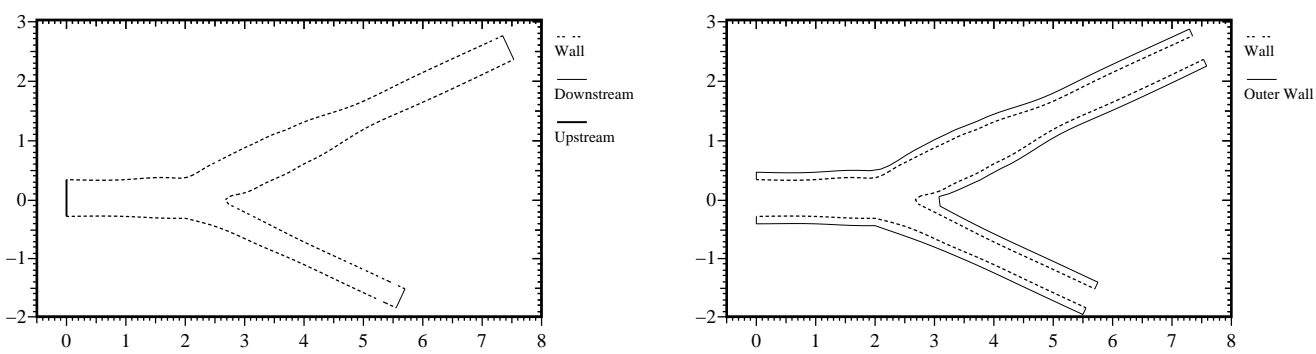

FIG. 5.1. A 2D model for the lumen (right) and the wall (left) of a carotid bifurcation. The dashed line represents the fluid-wall interface, the thick solid line represents the inflow of the lumen, while the thin solid line represents the outflow of the lumen or the outer surface of the arterial wall.

and the geometry are simplified (and in particular we deal with a 2D geometry obtained from data proposed in [7]), even if we include realistic numerical values for the physical constants at hand. In a forthcoming paper, on the basis of the good results illustrated in what follows, we plan to extend the methodology to more complex models and 3D geometries.

In the present section, as a realistic example, we apply our iterative substructuring method to the case of a carotid bifurcation, which is a preferential site of atherosclerosis, specifically in the external side of the carotid sinus. A current hypothesis about the possible reasons of this prominence to disease development is related to a reduction in the oxygen absorption by the sinus arterial wall, induced by the local flow patterns (see, e.g., [15]).

For the carotid test case we have considered stationary and pulsatile flow conditions. The selection of the boundary data is based on those proposed in [7]. In the stationary case, a parabolic profile is imposed on the upstream section, $u_{x}=$ $k u_{\max }\left(R^{2}-y^{2}\right), u_{y}=0$ where $k=10.4, u_{\max }=10 \mathrm{~cm} \mathrm{~s}^{-1}, R=0.31 \mathrm{~cm}$, while null velocity, $\mathbf{u}=0$, is prescribed on the wall boundary and zero traction force is prescribed on the outflow section, $(\rho \nu \nabla \mathbf{u}-P I) \cdot \mathbf{n}=0$ (see Figure 5.1 for a description of the boundaries $\partial \Omega_{f}$ and $\partial \Omega_{w}$ ). The pulsatile case differs from the stationary one for the inflow conditions, $u_{x}=k g(t)\left(R^{2}-y^{2}\right), u_{y}=0$, where $g(t)$, represented in Figure 5.2 , describes the blood flow at the entrance of the carotid during a heartbeat. In both cases, a constant blood kinematic viscosity $\nu=0.033 \mathrm{~cm}^{2} \mathrm{~s}^{-1}$ has been chosen.

With regard to the concentration in the lumen, a reference concentration of oxygen, $C_{0}=0.04 \mathrm{~g} \mathrm{~cm}^{-3}$, has been prescribed on the inflow, condition $\left(2.2_{3}\right)$ has been prescribed on the wall boundary, and $\nabla C \cdot \mathbf{n}=0$ has been prescribed on the outflow boundary. This condition is also prescribed on the outer wall in $\Omega_{w}$. The oxygen dynamics have been simulated choosing the diffusivity $\mu_{f}=\mu_{w}=10^{-5} \mathrm{~cm}^{2} \mathrm{~s}^{-1}$, and $\zeta=10^{-4}(1+|\boldsymbol{\sigma}|) \mathrm{cm} \mathrm{s}^{-1}$, where $\boldsymbol{\sigma}$ is the shear stress exerted by the blood on the wall. From the numerical viewpoint, we consider $\Omega_{f}$ discretized with 20494 nodes and $\Omega_{w}$ discretized with 15030 nodes (see Figure 5.3). With regard to boundary conditions, Dirichlet ones are imposed in an essential way, while Neumann and Robin conditions are imposed in a natural way, as is customary in the framework of Galerkin discretization and, in particular, in the framework of the FEM; see [12]. Finally, the numerical method adopted ensures a first order accuracy in time, being based on an implicit Euler scheme. The finite elements adopted are piecewise linear. The Navier-Stokes solver is based on the so-called Yosida method (see [10]) on $\mathbb{P}^{1}$ iso $\mathbb{P}^{2}$ elements.

Concerning the numerical results, the velocity field in the bifurcation shows a recirculation zone (see Figures 5.3 and 5.4) both in the steady and pulsatile cases. 

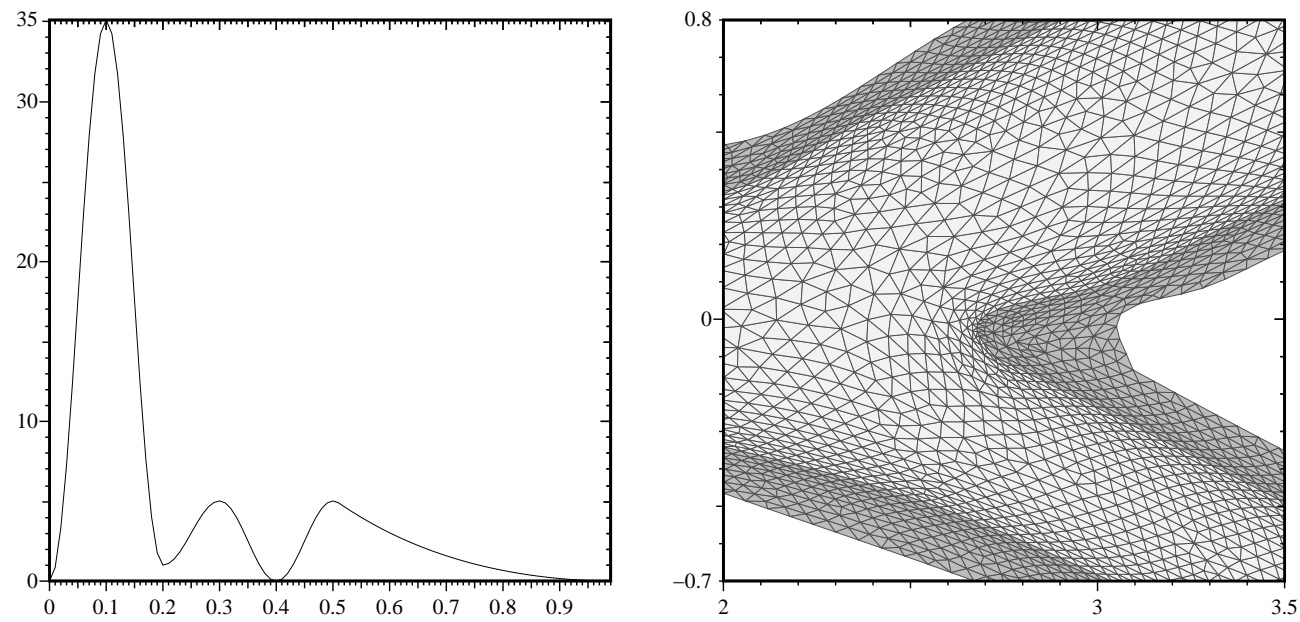

FIG. 5.2. Maximum inflow velocity $(\mathrm{cm} / \mathrm{s})$ during a heartbeat (left) and computational grid for the $2 D$ carotid simulation (right).
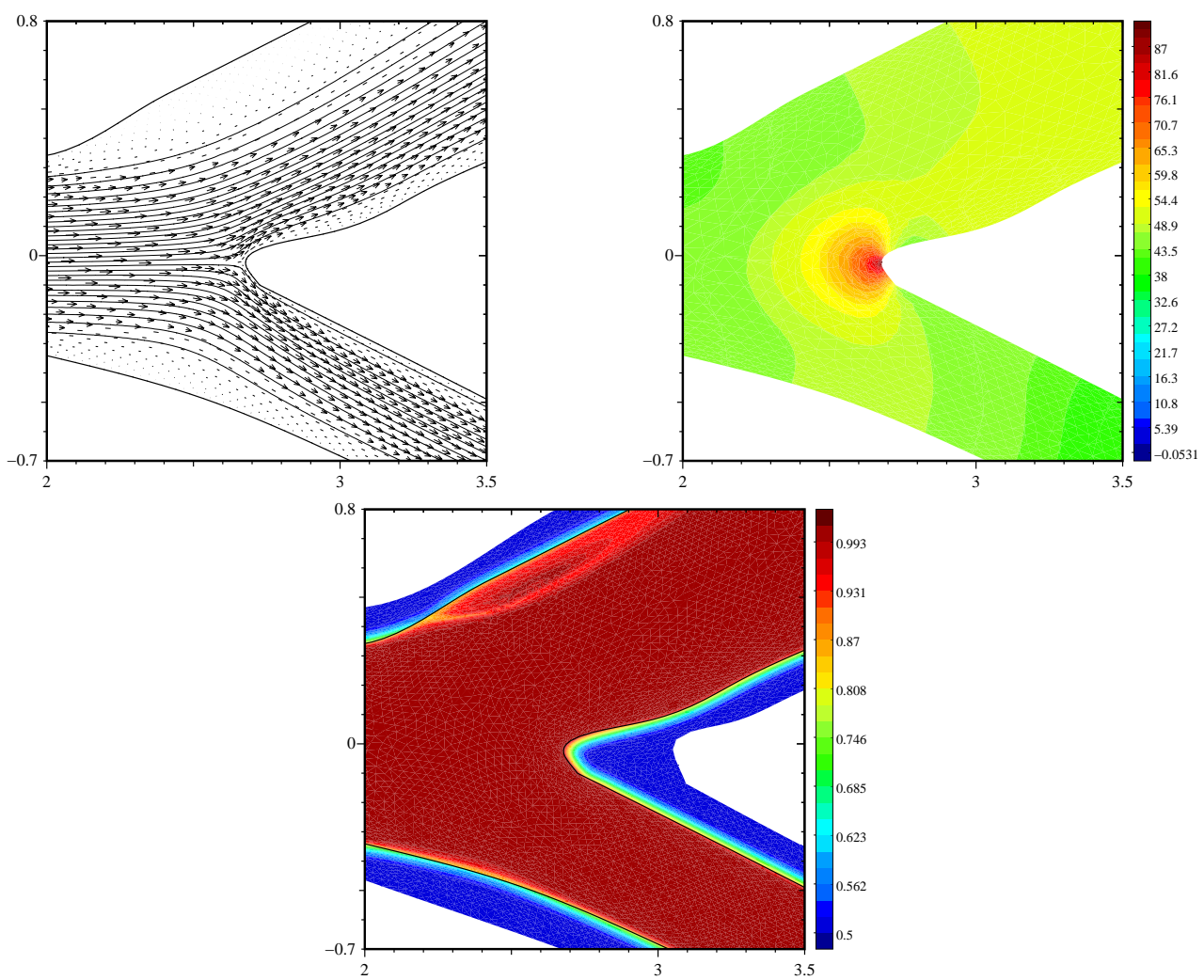

FIG. 5.3. Velocity field (top-left), pressure field $\left(P-P_{\text {ext }}\right)$ (top-right), and oxygen concentration (bottom) in the carotid bifurcation in the steady case. 

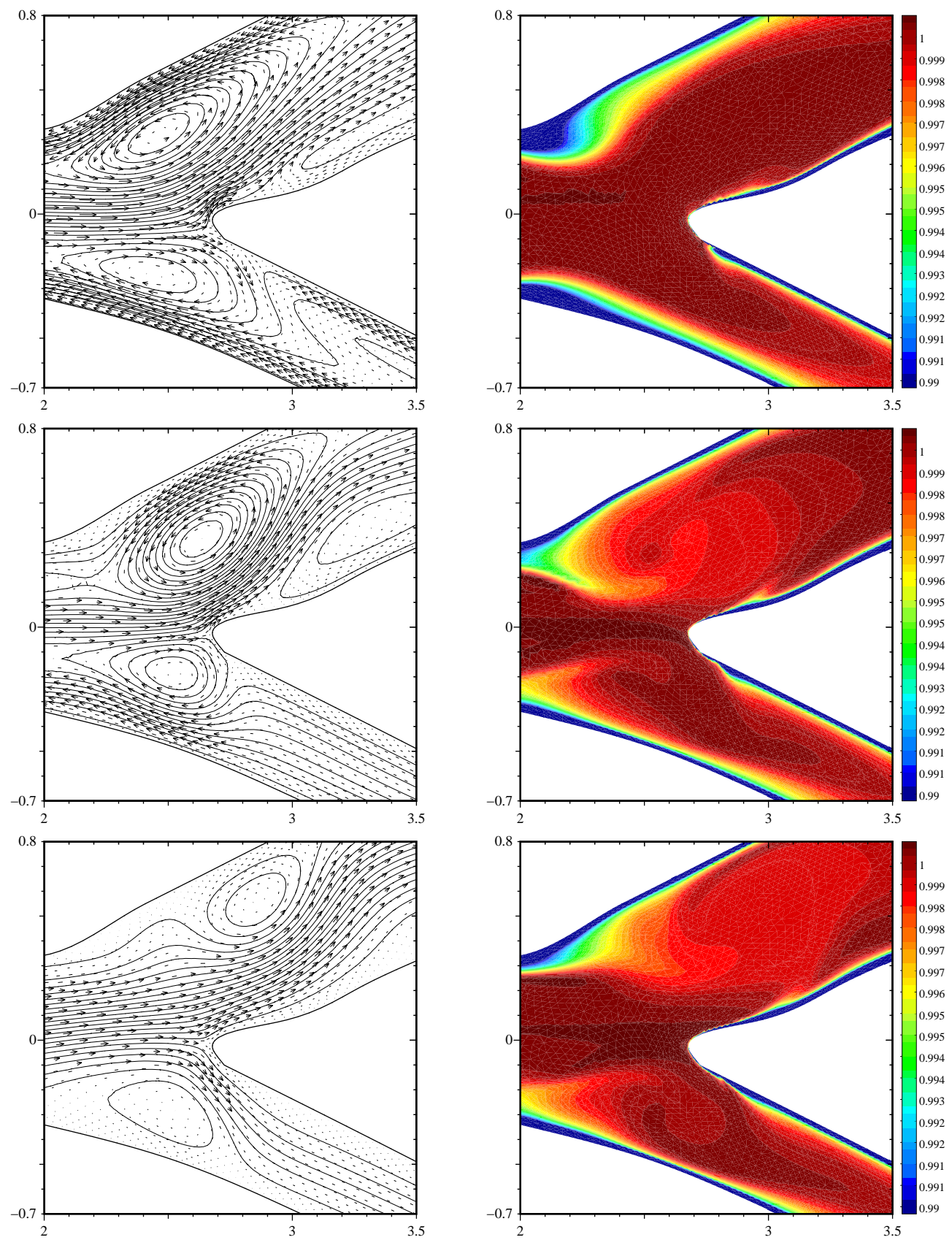

FIG. 5.4. Blood velocity and oxygen concentration in the carotid bifurcation in the pulsatile case at $1 / 5,2 / 5,3 / 5$ of a heartbeat. In this case only the concentration in $\Omega_{f}$ is visualized. The recirculation zone which possibly inhibits the oxygen absorption is evident in the different instants of the heartbeat.

The presence of recirculations induces in the steady case a sensibly lower oxygen concentration in proximity of the sinus wall and, consequently, the reduction of the oxygen absorption by the wall, which could be related to the disease development. 
In the pulsatile case the flow pattern is more complex: a recirculation zone, moving upstream and downstream (yielding a lack of oxygen), is still present next to the external side of the (inner) carotid sinus. In this case, the oxygen absorption is further reduced by the low permeability induced by the small values of $|\boldsymbol{\sigma}|$. Indeed, the flow dynamics in the carotid sinus induce small and oscillating shear stresses which actually determine a permeability reduction according to the law $\zeta=10^{-4}(1+|\boldsymbol{\sigma}|) \mathrm{cm} \mathrm{s}^{-1}$ (see also [18]).

6. Conclusions. In this paper we analyzed from the numerical viewpoint the solution of problem (2.2). The multidomain and the heterogeneous nature of this problem induced us to rely on iterative methods to compute the global solution. Consequently, in the framework of iterative substructuring methods, we introduced special SP operators in order to take into account conditions $\left(2.2_{3}, 2.2_{4}\right)$. The analysis of the properties of these operators allowed us to apply domain decomposition theory in order to prove the convergence of the iterative methods adopted to solve problem (2.2). Additionally, we proved that the mesh size does not affect the rate of convergence of the methods. Finally the problem of reducing the computational time was taken into account. To this end, we considered a variant of the GMRES algorithm, the so called F-P-GMRES, and we verified, with numerical tests, that this method is particularly efficient in solving the problems arising from the blood solute dynamics. The simplified test case considered, namely the carotid bifurcation, has been discussed from both the numerical and the physiological point of view.

\section{REFERENCES}

[1] C.G. Caro, J.M. Fitzgerald, And R.C. Schroter, Atheroma and wall shear: Observation, correlation and proposal of a shear dependent mass transfer mechanism of atherogenesis, Proc. Roy. Soc. London Ser. A, 177 (1971), pp. 109-159.

[2] G. Karner And K. PerkTold, Effect of endothelial injury and increased blood pressure on albumin accumulation in the arterial wall: A numerical study, J. Biomech., 33 (2000), pp. 709-715.

[3] G. Karner, K. Perktold, And H.P. Zehentner, Mass transport in large arteries and through the arterial wall, in Intra and Extracorporeal Cardiovascular Fluid Dynamics, WIT Press, Southampton, Boston, 2000, pp. 209-247.

[4] K.H. Keller, Effect of fluid shear on mass transport in flowing blood, Fed. Proc., 30 (1971), pp. 1591-1599.

[5] J.L. Lions and E. Magenes, Problèmes aux Limites non Homogènes et Applications, Vol. 1, Dunod, Paris, 1968.

[6] J.L. Lions and E. Magenes, Problèmes aux Limites non Homogènes et Applications, Vol. 2, Dunod, Paris, 1968.

[7] P. MA, X. Li, AND D.N. Ku, Convective mass transfer at the carotid bifurcation, J. Biomech., 30 (1997), pp. 565-571.

[8] J.A. Moore and C.R. Ethier, Oxygen mass transfer calculations in large arteries, J. Biomech. Engrg., 119 (1997), pp. 469-475.

[9] A. Quarteroni, R. Sacco, and F. Saleri, Numerical Mathematics, Springer-Verlag, New York, 2000.

[10] A. Quarteroni, F. Saleri, and A. Veneziani, Analysis of the Yosida method for the incompressible Navier-Stokes equations, J. Math. Pure Appl., 78 (1999), pp. 473-503.

[11] A. Quarteroni, M. Tuveri, And A. Veneziani, Computational vascular fluid dynamics: Problems, models and methods, Comput. Visual. Sci., 2 (2000), pp. 163-197.

[12] A. Quarteroni and A. Valli, Numerical Approximation of Partial Differential Equations, Springer-Verlag, Berlin, 1994.

[13] A. Quarteroni and A. Valli, Domain Decomposition Methods for Partial Differential Equations, Oxford University Press, Oxford, UK, 1999.

[14] A. Quarteroni, A. Veneziani, And P. Zunino, Mathematical and numerical modeling of solute dynamics in blood flow and arterial walls, SIAM J. Numer. Anal., 39 (2001), pp. 14881511. 
[15] G. Rappitsch And K. Perktold, Pulsatile albumin transport in large arteries: A numerical simulation study, J. Biomech. Engrg., 118 (1996), pp. 511-519.

[16] Y. SAAD, Iterative Methods for Sparse Linear Systems, PWS, Boston, 1996.

[17] R. Tемам, Navier-Stokes Equations. Theory and Numerical Analysis, 3rd ed., North-Holland, Amsterdam, 1984.

[18] C. Zarins, D. Giddens, B. Bharadvaj, V. Sottiurai, R. Mabon, and S. Glagov, Carotid bifurcation atherosclerosis: Quantitative correlation of plaque formation with velocity profiles and wall shear stresses, Circ. Res., 53 (1993), pp. 502-514. 